\title{
SIMULTANEOUS ATTAINABILITY OF CENTRAL LYAPUNOV AND BOHL EXPONENTS FOR ODE LINEAR SYSTEMS
}

\author{
ROBERT E. VINOGRAD
}

\begin{abstract}
Millionščikov's Accessibility Theorem for the central Lyapunov exponent of a linear ODE system is extended to simultaneous attainability of both central Lyapunov and Bohl exponents.
\end{abstract}

1. Let

$$
\dot{x}=A(t) x, \quad t \geqslant 0, x \in \mathbf{R}^{n},\|A(t)\| \leqslant a_{0} .
$$

The Lyapunov exponent $\lambda(x)$ and Bohl exponent $\beta(x)$ of a solution $x(t)$ are given by

$$
\lambda(x)=\varlimsup_{t \rightarrow \infty} \frac{1}{t} \ln |x(t)|, \quad \text { resp. } \beta(x)=\varlimsup_{t \rightarrow s \rightarrow \infty} \frac{1}{t-s} \ln \frac{|x(t)|}{|x(s)|} .
$$

(In fact these are upper exponents; the lower ones are defined similarly, with lim in place of $\overline{\lim }$.)

In general neither these exponents nor their suprema $\lambda_{0}=\sup _{x} \lambda(x), \beta_{0}=$ $\sup _{x} \beta(x)$ are stable under small perturbations of the system. Instead the so-called central Lyapunov exponent ${ }^{1} \Lambda \geqslant \lambda_{0}$ and Bohl exponent $B \geqslant \beta_{0}$ can be defined being stable upward (resp. lower exponents being stable downward). To introduce them and to describe exactly this "upward stability" we need a notion of upper functions (for brevity we omit similar notions and results about lower exponents).

2. Let $X(t, s)=X(t) X^{-1}(s)$ where $X(t)$ is a fundamental matrix of (1). As is known,

$$
|X(t, s)| \leqslant e^{a_{0}|t-s|}
$$

and

$$
|X(t, s)|=\max _{x} \frac{|x(t)|}{|x(s)|},
$$

where max is taken over all nonzero solutions of (1).

Definition. A bounded function $K(t)$ is an upper function for system (1) if there is a constant $D=D_{K}$ such that

$$
|X(t, s)| \leqslant D e^{\int_{s}^{t} K(\alpha) d \alpha} \quad(t \geqslant s) .
$$

Received by the editors November 17, 1982.

1980 Mathematics Subject Classification. Primary 34D05, 34D10.

${ }^{1}$ More popular notation is $\Omega$ rather than $\Lambda$. 
For example, by (2), $K(t)=a_{0}$ is an upper function with $D=1$. Let

$$
\bar{K}=\varlimsup_{t \rightarrow \infty} \frac{1}{t} \int_{0}^{t} K(\alpha) d \alpha, \quad \overline{\bar{K}}=\varlimsup_{t \rightarrow s \rightarrow \infty} \frac{1}{t-s} \int_{s}^{t} K(\alpha) d \alpha .
$$

Definition. The central Lyapunov exponent $\Lambda$, resp. Bohl exponent $\mathrm{B}$ is given by

$$
\Lambda=\inf \bar{K}, \quad \text { resp. } \mathrm{B}=\inf \overline{\bar{K}}
$$

where the inf is taken over all upper functions.

It is easily seen that $\lambda_{0} \leqslant \Lambda, \beta_{0} \leqslant \mathrm{~B}$ and $\Lambda \leqslant \mathrm{B}$.

3. Consider a perturbed system

$$
\dot{y}=[A(t)+\tilde{A}(t)] y
$$

and let its upper functions and exponents be marked by -

The upward stability of $K(t), \Lambda, \mathrm{B}$ means that given $\varepsilon>0$ there is $\delta=\delta(\varepsilon)>0$ such that if $|\tilde{A}(t)| \leqslant \delta$, then

$$
\tilde{K}(t) \leqslant K(t)+\varepsilon, \quad \tilde{\Lambda} \leqslant \Lambda+\varepsilon, \quad \tilde{\mathrm{B}} \leqslant \mathrm{B}+\varepsilon .
$$

The next theorem is well known [1].

4. TheOREM. $K(t), \Lambda$, and B are always upward stable.

Proof. It suffices to prove $\tilde{K}(t) \leqslant K(t)+\varepsilon$; then the rest follows by (5), (6). Let $Y(t, s)=Y(t) Y^{-1}(s)$ where $Y(t)$ is a fundamental matrix of (7). By the Variation of Constants Formula,

$$
Y(t, s)=X(t, s)+\int_{s}^{t} X(t, \tau) \tilde{A}(\tau) Y(\tau, s) d \tau .
$$

Take norms, use (4) and set

$$
|Y(t, s)|=D e^{\int_{s}^{t} K(\alpha) d \alpha} u(t) .
$$

Then

$$
u(t) \leqslant 1+\int_{s}^{t} D|\tilde{A}(\tau)| u(\tau) d \tau
$$

and by Gronwall's inequality, $u(t) \leqslant \exp \int_{s}^{t} D|A(\tau)| d \tau$. Now, if $|\tilde{A}(t)| \leqslant \delta$, then by $(8) \tilde{K}(t)=K(t)+D \delta$ is upper for (7). So $\delta(\varepsilon)=\varepsilon / D$.

In particular Theorem 4 implies that if $\lambda_{0}=\lambda$ (or $\beta_{0}=\mathrm{B}$ ), then $\lambda_{0}\left(\right.$ or $\left.\beta_{0}\right)$ is itself stable up. As is known, for a constant system (1) (i.e. $A(t)=$ const) one has always $\lambda_{0}=\beta_{0}=\Lambda=\mathrm{B}$, and so all exponents are stable up.

5. In contrast, for nonautonomous systems the central exponents $\Lambda$ and $\mathrm{B}$ need not be attainable by individual solution exponents, i.e. it may happen that $\lambda_{0}<\Lambda$ and/or $\lambda_{0}<\mathrm{B}$ (as well as $\Lambda<\mathrm{B}$ ). However the Accessibility Theorem [2] states that the central Lyapunov exponent $\Lambda$ is always attainable by means of arbitrarily small perturbations in the following sense: given $\delta>0$ there is a perturbation with $|\tilde{A}(t)|<\delta$ such that $\tilde{\lambda}_{0} \geqslant \Lambda$ for the perturbed system (7).

It turns out that this theorem can be extended to the attainability of B; moreover, a simultaneous attainability of both $\Lambda$ and $\mathrm{B}$ can be established and at the same time the original proof [2] can be considerably shortened. 
6. THEOREM. Let system (1) have central Lyapunov exponent $\Lambda$ and Bohl exponent B. Given $\delta_{0}>0$ there is a perturbation $\tilde{A}(t)$ with $|\tilde{A}(t)| \leqslant \delta_{0}$ such that system (6) has a solution $y(t)$ with both $\lambda(y) \geqslant \Lambda$ and $\beta(y) \geqslant \mathrm{B}$.

To prove this theorem we start with a technical remark and a number of lemmas.

7. Remark. All the above definitions of exponents or upper functions are given with continuously varying $t$ and $s$. But nothing will be changed if we replace them by discrete variables $t_{n}=n T, s_{m}=m T$, where $T>0$ is fixed and $m, n=1,2, \ldots$ This follows by the fact that by $(2),|X(t, s)| \leqslant e^{a_{0} T}=$ const as well as $|x(t)| /|x(s)| \leqslant$ $e^{a_{0} T}=$ const for $|t-s| \leqslant T$, so that any difference between continuous $t$ and discrete $t_{n} \leqslant t<t_{n+1}$ vanishes by taking $\varlimsup$ lim or else is absorbed by the constant $D$ in (4). In particular, $K(t)$ remains upper if (4) holds just for $t=t_{n}, s=s_{m}$.

8. Lemma. Let $T>0$ be fixed, $t_{n}=n T, J_{n}=\left[t_{n-1}, t_{n}\right], n=0,1, \ldots$ and

$$
\ln |X(t, s)|=f(t, s), \text { i.e., }|X(t, s)|=e^{f(t, s)} .
$$

Define a step function $K(t)$ by

$$
K(t) \equiv \lambda_{n}=\frac{1}{T} f\left(t_{n}, t_{n-1}\right) \quad \text { on } J_{n}, n=1,2, \ldots
$$

(the illegal "double definition" at $t=t_{n}$ can be neglected). Then $K(t)$ is an upper function and hence $\bar{K} \geqslant \Lambda, \overline{\bar{K}} \geqslant \mathrm{~B}$.

Proof. By (2), $K(t)$ is bounded: $|K(t)| \leqslant a_{0}$. Since $X(t, s)=X(t, r) X(r, s)$, we have $f(t, s) \leqslant f(t, r)+f(r, s)$, and since

$$
f\left(t_{k}, t_{k-1}\right)=\lambda_{k} T=\int_{t_{k-1}}^{t_{k}} K(\alpha) d \alpha
$$

we have for $t=t_{n}, s=t_{m}, n \geqslant m$,

$$
f(t, s) \leqslant \Sigma f\left(t_{k}, t_{k-1}\right)=\int_{s}^{t} K(\alpha) d \alpha, \text { i.e. }|X(t, s)| \leqslant e^{\int_{s}^{t} K(\alpha) d \alpha} .
$$

By Remark 7, $K(t)$ is upper.

The next several lemmas constitute so-called Millionščikov's Rotation Method. It can be found in [2], that is why we mostly restrict ourselves to some brief outlines of the proofs. Recall that the angle $\gamma=\Varangle(a, b)$ between two vectors $a, b \in \mathbf{R}^{n}$ is given by $\cos \gamma=a \cdot b /(|a| \cdot|b|), 0 \leqslant \gamma \leqslant \pi$.

9. Lemma. Let $a$ and $c$ be vectors in $R^{n}$ with $|a|=|c|$ and $\Varangle(a, c)=\gamma \neq 0, \pi$. Then there is a unitary operator $U(t): R^{n} \rightarrow R^{n}$ defined on a given interval $J^{*}$ : $t^{*} \leqslant t \leqslant t^{*}+T, T \geqslant 1$, such that

(i) $U\left(t^{*}\right)=I, U\left(t^{*}+T\right) a=c$,

(ii) $|U(t)-I|=\left|U^{-1}(t)-I\right| \leqslant \gamma$,

(iii) $\left|\dot{U}(t) U^{-1}(t)\right| \leqslant \gamma$.

SKETCH OF PROOF. Let $V(\omega): R^{n} \rightarrow R^{n}$ be the rotation by the angle $\omega$ from $a$ to $c$ in the 2-plane $P_{a c}$ spanned by $a, c$, and $V(\omega)=$ identity on the orthogonal complement to $P_{a c}$. Then $V(\omega)$ is unitary and in a proper orthonormal basis of $R^{n}$ (the two 
first elements in $P_{a c}$ ) the matrix of $V(\omega)$ is

$$
\operatorname{diag}\left\{\left(\begin{array}{cc}
\cos \omega & -\sin \omega \\
\sin \omega & \cos \omega
\end{array}\right), 1, \ldots, 1\right\} .
$$

Set $U(t)=V\left[\left(t-t^{*}\right) \gamma / T\right]$. Then (i) is clear and (ii), (iii) follow by direct computation.

10. Lemma. Let $x(t)$ be a solution of (1) considered on an interval $J^{*}=\left[t^{*}, t^{*}+T\right]$, $T \geqslant 1$. Next, let $x\left(t^{*}+T\right)=a$, and $c$ be a vector with $|c|=|a|$ and $\Varangle(a, c)=\gamma \neq$ $0, \pi$. Then there is a perturbation $\tilde{A}(t)$ with norm

$$
|\tilde{A}(t)| \leqslant \gamma(2 a+1)
$$

such that the perturbed system (7) has a solution $y(t)$ with

$$
y\left(t^{*}\right)=x\left(t^{*}\right) \text { and } y\left(t^{*}+T\right)=c \quad\left(\text { so that }\left|y\left(t^{*}+T\right)\right|=\left|x\left(t^{*}+T\right)\right|\right) .
$$

-Proof. Let $y(t)=U(t) x(t)$ where $U(t)$ is as in Lemma 9. Then clearly (11) holds. Next, $\dot{y}=U \dot{x}+\dot{U} x=\left(U A U^{-1}+\dot{U} U^{-1}\right) y=(A+\tilde{A}) y$ where $\tilde{A}=U A U^{-1}-A+$ $\dot{U} U^{-1}$. By Lemma 9, $\left|\dot{U} U^{-1}\right| \leqslant \gamma$ and

$$
\begin{aligned}
\left|U A U^{-1}-A\right| & \leqslant\left|U A\left(U^{-1}-I\right)\right|+|(U-I) A| \\
& \leqslant|U A| \cdot \gamma+\gamma|A| \\
& =2 \gamma|A| \quad(\text { since } U \text { is unitary, }|U A|=|A|) .
\end{aligned}
$$

Now (10) follows.

11. Lemma. Let $a, b, c$ be three coplanar vectors in $R^{n}$ such that $|a|=|b|=|c|$ and $0 \leqslant \gamma \leqslant \theta$ where $\gamma=\Varangle(a, c), \theta=\Varangle(a, b)$. Then $c=\alpha a+\beta b$ where

$$
\beta=\frac{\sin \gamma}{\sin \theta}>0 \text { and } \alpha=\frac{\sin (\theta-\gamma)}{\sin \theta}>0
$$

Proof is by direct computation.

12. Proof of Theorem 6. Choose first $\gamma$ and $T$ as follows. Let $\delta=\delta_{0} / 2$. Fix $\gamma$ with

$$
0<\gamma \leqslant \delta /(2 a+1) .
$$

Then fix $T \geqslant 1$ and so large that $\sin \gamma \geqslant 2 e^{-\delta T}$, i.e.

$$
\sin \gamma-e^{-\delta T} \geqslant e^{-\delta T} .
$$

Define $K(t)$ as in Lemma 8 and classify the solutions $x(t)$ of system (1) on each interval $J_{n}=\left[t_{n-1}, t_{n}\right]$ as follows.

If

$$
\frac{\left|x\left(t_{n}\right)\right|}{\left|x\left(t_{n-1}\right)\right|} \begin{cases}=e^{\lambda_{n} T}, & \text { then } x(t) \text { is maximal on } J_{n}, \\ \geqslant e^{\left(\lambda_{n}-\delta\right) T}, & \text { then } x(t) \text { is rapid on } J_{n}, \\ <e^{\left(\lambda_{n}-\delta\right) T}, & \text { then } x(t) \text { is slow on } J_{n} .\end{cases}
$$

Notice that a maximal solution always exists by (3) and (9). Since a constant multiple of $x(t)$ falls into the same category as $x(t)$, we can normalize $x(t)$ as we like without change of its category. 
Now we are going to perturb system (1) inductively on each interval $J_{1}, J_{2}, \ldots$ Each time the perturbation $\tilde{A}(t)$ will be found by Lemma 10 and hence with $|\tilde{A}(t)| \leqslant \delta$ by virtue of (10) and (13). We will not mention this smallness any longer. Starting with a rapid solution on $J_{n}$ we will watch its behavior on $J_{n+1}$ and depending on that choose a perturbation on $J_{n}$ (but not on $J_{n+1}$ yet).

lst step. Pick a maximal solution $x(t)$ on $J_{1}$. Then it is also rapid

$$
\frac{\left|x\left(t_{1}\right)\right|}{\left|x\left(t_{0}\right)\right|}=e^{\lambda_{1} T}>e^{\left(\lambda_{1}-\delta\right) T} \quad\left(t_{0}=0\right) \text {. }
$$

Look at its natural extension to $J_{2}$. If it remains rapid on $J_{2}$, i.e.

$$
\frac{\left|x\left(t_{2}\right)\right|}{\left|x\left(t_{1}\right)\right|} \geqslant e^{\left(\lambda_{2}-\delta\right) T}
$$

then put $\tilde{A}(t) \equiv 0$ on $J_{1}$, relable $x(t)$ by.$l(t)$ on $J_{1}$, and the 1 st step is completed. As a result we have

$$
\frac{\left|y\left(t_{1}\right)\right|}{\left|y\left(t_{0}\right)\right|} \geqslant e^{\left(\lambda_{1}-\delta\right) T}, \quad \frac{\left|x\left(t_{2}\right)\right|}{\left|x\left(t_{1}\right)\right|} \geqslant e^{\left(\lambda_{2}-\delta\right) T}
$$

where $x(t)$ is a natural (unperturbed) extension of $y(t)$.

Suppose $x(t)$ is slow on $J_{2}$ and let $x\left(t_{1}\right)=a$. Find a maximal solution $\xi(t)$ on $J_{2}$ and normalize it so that the vector $\xi\left(t_{1}\right)=b$ has norm $|b|=|a|$. Since $x(t)$ is slow while $\xi(t)$ is maximal, they cannot be proportional; therefore $\Varangle(a, b) \neq 0, \pi$. Define a vector $c$ like this: $c=b$ if $\Varangle(a, b) \leqslant \gamma$, otherwise let $c=\alpha a+\beta b$ be as in Lemma 11.

Now perturb system (1) on $J_{1}$ as in Lemma 10. This yields a solution $y(t)$ of (7) with $y\left(t_{0}\right)=x\left(t_{0}\right),\left|y\left(t_{1}\right)\right|=\left|x\left(t_{1}\right)\right|$ and hence with

$$
\frac{\left|y\left(t_{1}\right)\right|}{\left|y\left(t_{0}\right)\right|} \geqslant e^{\left(\lambda_{1}-\delta\right) T}
$$

The crucial point is that the natural (unperturbed) extension $z(t)$ of $y(t)$ beyond $t_{1}$ is rapid on $J_{2}$. Indeed, if $c=b$, then $z(t)=\xi(t)$ which is even maximal on $J_{2}$. Otherwise $z\left(t_{1}\right)=c=\alpha a+\beta b=\alpha x\left(t_{1}\right)+\beta \xi\left(t_{1}\right)$ and by linearity

$$
z(t)=\alpha x(t)+\beta \xi(t), \quad t \geqslant t_{1} .
$$

At $t=t_{1}$ all three norms of $z, x, \xi$ are equal, therefore

$$
\frac{\left|z\left(t_{2}\right)\right|}{\left|z\left(t_{1}\right)\right|}=\frac{\left|\alpha x\left(t_{2}\right)+\beta \xi\left(t_{2}\right)\right|}{\left|\xi\left(t_{1}\right)\right|} \geqslant \frac{\left|\xi\left(t_{2}\right)\right|}{\left|\xi\left(t_{1}\right)\right|}\left(\beta-\alpha \frac{\left|x\left(t_{2}\right)\right|}{\left|\xi\left(t_{2}\right)\right|}\right) .
$$

Since $\xi$ is maximal and $x$ is slow on $J_{2}$, we have

$$
\frac{\left|x\left(t_{2}\right)\right|}{\left|\xi\left(t_{2}\right)\right|}=\frac{\left|x\left(t_{2}\right)\right| /\left|x\left(t_{1}\right)\right|}{\left|\xi\left(t_{2}\right)\right| /\left|\xi\left(t_{1}\right)\right|}<\frac{e^{\left(\lambda_{2}-\delta\right) T}}{e^{\lambda_{2} T}}=e^{-\delta T} .
$$


Then

$$
\begin{aligned}
\beta-\alpha \frac{\left|x\left(t_{2}\right)\right|}{\left|\xi\left(t_{2}\right)\right|} & >\frac{\sin \gamma-\sin (\theta-\gamma) e^{-\delta T}}{\sin \theta} \\
& \geqslant \sin \gamma-e^{-\delta T} \\
& \geqslant e^{-\delta T} \quad \text { (by (14)). }
\end{aligned}
$$

Combining with (15), $z$ is rapid on $J_{2}$

$$
\frac{\left|z\left(t_{2}\right)\right|}{\left|z\left(t_{1}\right)\right|} \geqslant e^{\left(\lambda_{2}-\delta\right) T} .
$$

Relabeling $z(t)$ by $x(t)$ on $J_{2}$, we come again to (15), and the 1 st step is entirely completed.

Suppose we have already completed $m-1$ steps of the induction with the following results:

(i) The system is properly perturbed on $J_{1} \cup \cdots \cup J_{m-1}$ but unperturbed yet on $J_{m}=\left[t_{m-1}, t_{m}\right]$ or further.

(ii) There is a solution $y(t)$ of the perturbed system on $J_{1} \cup \cdots \cup J_{m-1}$ with natural (unperturbed) continuous extension $x(t)$ on $J_{m}$ such that

$$
\begin{gathered}
\frac{\left|y\left(t_{k}\right)\right|}{\left|y\left(t_{k-1}\right)\right|} \geqslant e^{\left(\lambda_{k}-\delta\right) T}, \quad k=1, \ldots, m-1, \\
\frac{\left|x\left(t_{m}\right)\right|}{\left|x\left(t_{m-1}\right)\right|} \geqslant e^{\left(\lambda_{m}-\delta\right) T} .
\end{gathered}
$$

$m$ th step is now exactly as the 1st one, just with $t_{m-1}, t_{m}, t_{m+1}$ in place of $t_{0}, t_{1}, t_{2}$. Namely, if $x(t)$ remains rapid on $J_{m+1}$ too, then we set $\tilde{A}(t) \equiv 0$ on $J_{m}$, relabel $x(t)$ by $y(t)$ on $J_{m}$ and so get $(17 \mathrm{a}, \mathrm{b})$ with $m$ replaced by $m+1$. In this case the $m$ th step is completed.

If $x(t)$ is slow on $J_{m+1}$, then let $x\left(t_{m}\right)=a$, find a maximal solution $\xi(t)$ on $J_{m+1}$ with $\xi\left(t_{m}\right)=b,|b|=|a|$, and define $c$ as before: $c=b$ if $\Varangle(a, b) \leqslant \gamma$, otherwise $c=\alpha a+\beta b$ as in Lemma 11. Now perturb the system on $J_{m}$ as in Lemma 10. This creates a solution $y(t)$ with $y\left(t_{m-1}\right)=x\left(t_{m-1}\right),\left|y\left(t_{m}\right)\right|=\left|x\left(t_{m}\right)\right|$ and hence, by (17b), with

$$
\frac{\left|y\left(t_{m}\right)\right|}{\left|y\left(t_{m-1}\right)\right|} \geqslant e^{\left(\lambda_{m}-\delta\right) T} .
$$

As before, the unperturbed continuous extension $z(t)$ of $y(t)$ beyond $t_{m}$ is rapid on $J_{m+1}$, and relabeling $z(t)$ by $x(t)$ gives again $(17 \mathrm{a}, \mathrm{b})$ with $m$ replace by $m+1$. The $m$ th step is entirely completed.

By induction, we obtain a system (7) defined for all $t \geqslant 0$ with perturbation $\tilde{A}(t)$ of smallness $|\tilde{A}(t)| \leqslant \delta=\delta_{0} / 2$ and having a solution $y(t)$ which satisfies (17a) for all $k=1,2, \ldots$ By the very definition (9) of $K(t)$,

$$
\int_{t_{n-1}}^{t_{n}} K(\alpha) d \alpha=\lambda_{n} T, \quad \int_{t_{n-1}}^{t_{n}}[K(\alpha)-\delta] d \alpha=\left(\lambda_{n}-\delta\right) T
$$


so that (17a) implies for $s=t_{m}, t=t_{n}(t \geqslant s)$

$$
\frac{|y(t)|}{|y(0)|} \geqslant \exp \int_{0}^{t}[K(\alpha)-\delta] d \alpha \text { and } \frac{|y(t)|}{|y(s)|} \geqslant \exp \int_{s}^{t}[K(\alpha)-\delta] d \alpha .
$$

It follows by Remark 7 and Lemma 8 that the Lyapunov and Bohl exponents of $y(t)$ satisfy $\lambda(y) \geqslant \bar{K}-\delta \geqslant \Lambda-\delta$ and $\beta(y) \geqslant \overline{\bar{K}}-\delta \geqslant \mathrm{B}-\delta$ respectively. To complete the proof let $y^{*}(t)=y(t) e^{\delta t}$. Then $\lambda\left(y^{*}\right) \geqslant \Lambda, \beta\left(y^{*}\right) \geqslant \mathrm{B}$ and $y^{*}(t)$ satisfies the system with perturbation $\tilde{A}(t)+\delta I$ of smallness $2 \delta=\delta_{0}$.

\section{REFERENCES}

1. B. F. Bylov, D. M. Grobman, V. V. Nemyckiǐ and R. E. Vinograd, The theory of Lyapunov exponents, "Nauka", Moscow, 1966, pp. 164-166. (Russian)

2. V. M. Millionščikov, A proof of accessibility of central exponents of linear systems, Sibirsk Mat. Ž. 10 (1969), 99-104. (Russian)

Department of Mathematical Sciences, North Dakota State University, Fargo, North DAKOTA 58105 